\title{
Projeto terapêutico singular: uma ferramenta de promoção da saúde do idoso
}

\author{
Unique therapeutic project: a tool to promote the health of the elderly
}

Procedimiento simple: la herramienta para promover la salud de los ancianos

Francisca Thamires Lima de Sousa ${ }^{1 *}$, Kezia Cristina Batista dos santos ${ }^{2}$, Shirley Barbosa Silva ${ }^{2}$, Arleane Debora dos Santos Gonçalves², Camila Jane Mota Pereira².

\section{RESUMO}

Objetivo: Relatar a experiência de residentes multiprofissionais em Saúde do Adulto e Idoso sobre a elaboração do Projeto Terapêutico Singular (PTS) no Hospital Universitário da Universidade Federal do Maranhão. Detalhamento do caso: A residência multiprofissional em saúde promove a interdisciplinaridade do cuidado pelo trabalho conjunto de diferentes categorias profissionais nas atividades assistenciais e de educação em saúde. O PTS consiste em um instrumento de ação baseado na articulação de condutas terapêuticas para um sujeito resultante da discussão coletiva da equipe interdisciplinar. Durante o treinamento em serviço os residentes participaram de reuniões, rodas de conversa e atividades de planejamento e execução do PTS voltados para resolução de casos complexos na assistência à saúde de idosos em situação de vulnerabilidade. Foram realizados encontros semanais para discussão dos casos, visando alinhar os objetivos terapêuticos a curto, médio e longo prazo, resultando na construção do plano de intervenções baseado nas necessidades de saúde, sociais e político-programáticas dos casos. Considerações finais: Verificou-se que a proposta do PTS permitiu a problematização por parte da equipe multiprofissional das necessidades específicas do idoso, contemplando a integralidade da atenção em uma perspectiva humanizada do atendimento, socialização dos saberes, incorporação da multidimensionalidade nas abordagens e $\mathrm{o}$ aprimoramento das práticas assistenciais.

Palavras-chave: Assistência à Saúde, Equipe de Assistência ao Paciente, Integralidade em Saúde.

\begin{abstract}
\footnotetext{
1Universidade Estadual do Maranhão (UEMA). São Luís-MA. *E-mail: assts.tham@gmail.com.

2 Universidade Federal do Maranhão (UFMA). São Luís-MA.

${ }^{2}$ Programa de Pós-graduação em Políticas Públicas. São Luís - MA.

2 Especialista em Saúde com ênfase na Atenção à Saúde da Mulher. São Luís-MA.

${ }^{2}$ Especialista em saúde com ênfase na Atenção Cardiovascular. São Luís-MA.
}

Objective: To report the experience of multiprofessional residents in Adult and Elderly Health on the elaboration of the Unique Therapeutic Project (PTS) at the University Hospital of the Federal University of Maranhão. Case detail: Multiprofessional health residency promotes the interdisciplinarity of care by the joint work of different professional categories in healthcare and health education activities. The PTS consists of an instrument of action based on the articulation of therapeutic behaviors for a subject resulting from the collective discussion of the interdisciplinary team. During on-the-job training, residents attended meetings, talk wheels, and planning and implementation activities of the PTS aimed at resolving complex cases in the health care of vulnerable elderly. Weekly meetings were held to discuss the cases, aiming to align the therapeutic objectives in the short, medium and long term, resulting in the construction of the intervention plan based on the health, social and political-programmatic needs of the cases. Final considerations: It was verified that the PTS proposal allowed the multiprofessional team to question the specific needs of the elderly, contemplating the 
integrality of attention in a humanized perspective of care, socialization of knowledge, incorporation of multidimensionality in approaches and improvement of practices.

Key words: Delivery of Health Care, Patient Care Team, Integrality in Health.

\section{RESUMEN}

Objetivo: Informar la experiencia de residentes multiprofesionales en Salud del Adulto y Anciano sobre la elaboración del Proyecto Terapéutico Singular (PTS) en el Hospital Universitario de la Universidad Federal de Maranhão. Detalle del caso: La residencia multiprofesional en salud promueve la interdisciplinariedad del cuidado por el trabajo conjunto de diferentes categorías profesionales en las actividades asistenciales y de educación en salud. EI PTS consiste en un instrumento de acción basado en la articulación de conductas terapéuticas para un sujeto resultante de la discusión colectiva del equipo interdisciplinario. Durante el entrenamiento en servicio los residentes participaron en reuniones, ruedas de conversación y actividades de planificación y ejecución del PTS orientados a la resolución de casos complejos en la asistencia a la salud de ancianos en situación de vulnerabilidad. Se realizaron encuentros semanales para discusión de los casos, con el objetivo de alinear los objetivos terapéuticos a corto, medio y largo plazo, resultando en la construcción del plan de intervenciones basado en las necesidades de salud, sociales y político-programáticas de los casos. Consideraciones finales: Se verificó que la propuesta del PTS permitió la problematización por parte del equipo multiprofesional de las necesidades específicas del anciano, contemplando la integralidad de la atención desde una perspectiva humanizada de la atención, socialización de los saberes, incorporación de la multidimensionalidad en los enfoques y el perfeccionamiento prácticas asistenciales.

Palabras clave: Prestación de Atención de Salud, Grupo de Atención al Paciente, Integralidad en Salud.

\section{INTRODUÇÃO}

A população global vem vivenciando um processo continuo de envelhecimento, em função da queda da fecundidade e da mortalidade. Os idosos com 60 anos ou mais no mundo, alcançou 770 milhões de pessoas em 2010, cerca de $11 \%$ da população. Esse número deve chegar a 1 bilhão em 2020 (aproximadamente 13\% da população mundial), entre eles $20 \%$ residirem em países em desenvolvimento a exemplo do Brasil (CENSUS BUREAU US, 2016).

Em 2001 o Sistema de Informação Hospitalar do Sistema Único de Saúde (SIHSUS) registrou 12.227.465 internações hospitalares no âmbito do Sistema Único de Saúde. Os idosos, que representavam 8,5\% da população geral, corresponderam a 18,3\% das hospitalizações (CUNHA BSS, 2013).

Nesta perspectiva, a população idosa tem grande impacto no uso dos serviços de saúde e apresenta elevadas taxas de internação, além de maior tempo médio de ocupação do leito hospitalar. Segundo dados do Departamento de Informações do Sistema Único de Saúde (DATASUS), a taxa de internação para a faixa de idade acima de 60 anos está em torno de 242,2 internações por 1.000 hab./ano, representando o triplo da taxa de internação para faixa dos 15 aos 39 anos, e o tempo médio de permanência é o dobro quando comparado à mesma faixa etária, consumindo $26 \%$ de todos os recursos financeiros destinados à saúde (BRASIL, 2017).

Desse modo, estudos revelam que os idosos que apresentam alguma patologias crônico-degenerativas tem maior probabilidade de serem internados, além do que, eles gastam mais com os serviços de saúde, pois essas enfermidades elevam as taxas de internação (REIS CS, et al., 2016).

Diante desse cenário, a Residência Multiprofissional em Saúde (RMS) foi instituída no Sistema Único de Saúde (SUS) a fim de promover uma assistência integral e qualificada por meio de uma atenção interdisciplinar pela integração de diferentes profissões nos diversos cenários da saúde. Trata-se de uma modalidade de pós-graduação lato sensu, caracterizado por ensino em serviço com duração de 02 anos e 
carga horária total de 5.760 horas, $80 \%$ desta é destinada a atividades práticas comumente conhecida como treinamento em serviço (TS) e $20 \%$ para as atividades teóricas. Esta é destinada às profissões da saúde como: Serviço Social, Enfermagem, Farmácia, Fonoaudiologia, Fisioterapia, Nutrição, Odontologia, Educação Física, Terapia Ocupacional e Psicologia (BRASIL, 2009).

Perante o exposto, a escolha deste tema foi assentada a partir de discussões entre equipe interdisciplinar acerca da importância do PTS para prática assistencial na promoção da saúde do idoso, no intuito de orientar sua execução e minimizar possíveis dificuldades na conciliação entre teoria e prática, além de possibilitar intervenção multi e interdisciplinar em casos específicos de situações complexas e/ou de vulnerabilidades encontradas entre os pacientes idosos hospitalizados no campo de estudo.

O PTS se caracteriza como conjunto de propostas de condutas terapêuticas articuladas, para um sujeito individual ou coletivo, resultado da discussão coletiva de uma equipe interdisciplinar, com apoio matricial se necessário. Geralmente é dedicado a situações mais complexas. Compreende quatro momentos: 1. Diagnóstico; 2. Definição de metas; 3. Divisão de responsabilidades e; 4. Reavaliação (BRASIL, 2007).

Ressalta-se que, muito se fala das atividades em equipe multidisciplinar, mas poucos compartilham sua experiência durante esse processo. Diante disto, esse estudo objetivou relatar a experiência de residentes multiprofissionais em Saúde do Adulto e Idoso sobre a elaboração do Projeto Terapêutico Singular (PTS) no Hospital Universitário da Universidade Federal do Maranhão.

\section{DETALHAMENTO DO CASO}

Trata-se de um estudo descritivo, do tipo relato de experiência, realizado no período de 2016 a 2018, que apresenta a vivência de residentes multiprofissionais (assistentes sociais, enfermeiros, fisioterapeutas, terapeutas ocupacionais, farmacêuticos, fonoaudiólogos, nutricionistas, educadores físicos, odontólogos), sobre a elaboração do PTS na Unidade de Cuidados Clínicos do Adulto do Hospital Universitário da Universidade Federal do Maranhão. A base metodológica utilizada para a construção deste relato foi a Teoria de Intervenção Práxica de Enfermagem em Saúde Coletiva, estudos bibliográficos, documentais e a observação participante a partir de discussões, reuniões, rodas de conversa e atividades de planejamento e execução do PTS.

O local do estudo consiste em um hospital público de ensino, pesquisa e extensão localizado na cidade de São Luís do Maranhão, cuja oferta dos serviços de saúde à população usuária se dá no âmbito do Sistema Único de Saúde (SUS). Trata-se de um hospital federal de alta complexidade e que tem contribuído com a formação de diversos profissionais na área da saúde por exercer a função de hospital-escola.

O PTS é reconhecido como uma ferramenta de otimização dos recursos, sejam eles, humanos, materiais ou financeiros e também para a cogestão do processo terapêutico do idoso em situação de vulnerabilidade. Esta ferramenta foi implantada inicialmente nas Unidades de Cuidados Clínicos e Cirúrgicos do Adulto por possuírem um número elevado de pacientes em situação de vulnerabilidade durante o processo de hospitalização/recuperação.

As reuniões para operacionalização do PTS na instituição ocorreram semanalmente, contando com a participação dos preceptores, residentes em treinamento em serviço no setor e equipe assistencial. A equipe multiprofissional foi composta por médicos, enfermeiros, fisioterapeutas, técnicos de enfermagem, psicólogos, assistentes sociais, terapeutas ocupacionais, nutricionistas e fonoaudiólogos. As reuniões eram realizadas a beira leito, no posto de enfermagem e na secretaria da unidade para discussão dos casos e elaboração do PTS.

A escolha dos pacientes idosos seguiu critérios específicos de avaliação de riscos e vulnerabilidades, tentando captar a singularidade do sujeito em todos os seus aspectos biopsicossociais, dentre os quais podese citar: mínimo 10 dias de internação, complicações no quadro clínico, problemas sociais ou com acompanhante, dificuldade de interação com a equipe, resistência para realizar os procedimentos 
terapêuticos ou baixa adesão ao tratamento. Após a identificação do paciente e seleção do caso, uma reunião era realizada entre equipe interdisciplinar, paciente e/ou acompanhante para discussão e reflexão dos principais problemas encontrados e compreensão do contexto.

Observou-se que a maioria dos casos se tratava de idosos institucionalizados em situação de fragilidade que por alguma descompensação clínica encontravam-se internados na unidade. Destaca-se que muitos idosos não tinham apoio familiar ou social necessário, o que pode comprometer ainda mais sua qualidade de vida, já prejudicada pelo processo de senilidade enfrentada.

Posteriormente à identificação dos casos, nas reuniões seguintes, foram traçados objetivos e metas de curto, médio e longo prazo negociadas com o paciente e seu familiar sempre garantindo a integralidade do cuidado. Na próxima etapa, os profissionais dividiam suas responsabilidades de acordo com as propostas terapêuticas contidas no plano de intervenção estabelecido em equipe. O processo de avaliação do plano de intervenção era realizado semanalmente entre equipe interdisciplinar sempre na presença do paciente e/ou acompanhante para que fosse avaliado o real alcance dos objetivos e para que contasse com a participação dos mesmos nas decisões e intervenções realizadas.

É notável destacar que, essa ferramenta do cuidado integra teoria e prática na qual os profissionais envolvidos buscam alternativas eficazes e seguras para o desenvolvimento do PTS junto ao paciente e familiar. Observou-se que é necessário adaptação da teoria a realidade social do indivíduo e ao meio no qual ele está inserido. O sujeito nunca pode ser considerado de forma isolado pela equipe, sendo também coparticipantes seus familiares ou acompanhantes. A equipe buscou sempre atuar em prol da criação de vínculo terapêutico e promoção da escuta qualificada.

Destaca-se, que a cada reunião eram realizados os registros das avaliações do PTS, assim como metas e objetivos alcançados e anexados ao prontuário do paciente. Esse registro é muito importante pois traz informações relevantes sobre o PTS, assim como o histórico do paciente, dados de seu quadro clínico e identificação pessoal deste e de seus familiares e ou acompanhantes. É de suma importância destacar, que todas as informações são sigilosas, se mantendo ao alcance apenas da equipe responsável pelo caso, em respeito a legislação vigente e normas e princípios da Política Nacional de Atenção a Pessoa Idosa. Assim os residentes puderam apreender a multidimensionalidade da situação de saúde ao entender que esta não se limita apenas ao processo biológico e físico apresentado pelo paciente, mas que também está relacionada as dimensões psicológicas, sociais, culturais, econômicas, enfim, ao contexto em que o paciente se encontra inserido.

Ressalta-se que, atualmente quando um paciente recebe alta, este mantém um vínculo com a equipe. Os profissionais, em alguns casos, tentam manter contato com a rede de referência e contrarreferência pra receber um feedback dos encaminhamentos realizados.

Pode-se afirmar que a implementação do PTS na unidade impactou positivamente na qualidade dos idosos atendidos, uma vez que, eram discutidas as necessidades de saúde e biopsicossociais do caso entre equipe multiprofissional buscando-se soluções possíveis e de acordo com o contexto vivenciado pelo idoso dentro e fora do ambiente hospitalar. Os idosos que participaram do PTS sentiram-se seguros quanto à assistência prestada e mais confiantes e empoderados quanto ao seu tratamento reconhecendo seu papel dentro do contexto terapêutico do cuidado.

\section{DISCUSSÃO}

A Rede de Atenção à Saúde (RAS) no âmbito do SUS pode ser definida como um arranjo de ações e de serviços de saúde, de diferentes densidades tecnológicas, que integradas por meio de um conjunto de sistemas de apoio técnico, logístico e de gestão, buscam garantir a integralidade do cuidado. Seu principal objetivo é possibilitar a integração sistêmica de ações e de serviços em saúde (BRASIL, 2018).

Assim, ao se guiar pela RAS, o Ministério da Saúde lançou em 2014, um documento chamado: diretrizes para o cuidado das pessoas idosas no SUS, e definiu entre suas estratégias a assistência a esses pacientes 
com foco no Modelo de Atenção Integral. Suas diretrizes também definem parâmetros para o trabalho em equipes interprofissionais e multiprofissionais, além de ações intersetoriais (COELHO LP, et al., 2018).

Esse novo modelo de atenção integral a saúde do idoso, buscou promover recursos capazes de viabilizar uma assistente contínua com foco nos aspectos biopsicossociais do paciente de forma que ele seja visto em sua integralidade e não apenas como um indivíduo com uma determinada patologia, devendo este também opinar em seu tratamento quando a par de suas faculdades mentais e decidir com a equipe qual melhor terapêutica a ser implementada e seguida conforme seu contexto econômico e social.

Em vista disso, a Política Nacional de Humanização do Sistema Único de Saúde (HumanizaSUS) recomenda o uso do PTS como dispositivo que auxilia a transição da clínica tradicional médico-biologista para a clínica ampliada humanizada, articulando a tríade equipe-usuário-família, bem como construindo estratégias que visam a produção do cuidado e a assistência integral ao usuário (BRASIL, 2010).

As propostas de um PTS devem resultar de uma discussão coletiva do caso e dos vários olhares e saberes sobre a situação (BRASIL, 2010). Oliveira GN (2007) destaca ser fundamental a articulação entre as diversas especialidades profissionais, caracterizadas pelos distintos saberes e práticas, distintas visões sobre a problemática, de modo a organizar proposições comuns que expliquem o problema, permitindo à equipe a composição de estratégias de intervenção que vão garantir maior sucesso e menores riscos de agravo.

Aliado a isto, a Política Nacional HumanizaSUS acrescenta que tanto o paciente como seus familiares também devem participar e estar junto à equipe no processo de planejamento do PTS com intuito de promover condições de co-elaboração (BRASIL, 2007).

De acordo com Boccardo ACS et al. (2011) a construção do PTS requer a transformação do modelo assistencial e a reorganização dos serviços, pois a forma como estes são organizados pode facilitar ou dificultar a construção das estratégias previstas para a efetivação do PTS.

O processo de reorganização do trabalho em equipe e a construção de uma nova cultura assistencial deve também enfrentar o desafio de superar as normas e estruturas organizacionais tradicionais e introduzir uma nova forma divisão das responsabilidades, pautada na continuidade das ações e não mais na produção de respostas isoladas, imediatistas e descontextualizadas (BARROS JO, 2009; BOCCARDO ACS, et al., 2011).

Compreende-se que o processo saúde-doença é dinâmico, que ora demanda uma intervenção mais focada nos aspectos biológicos ou psicossociais e ora requisita maior integração entre esses aspectos. Contudo, sempre será preciso transformar o saber sobre o padrão das doenças em um saber sobre a pessoa doente, considerando sua singularidade e toda a imprevisibilidade e as contradições inerentes ao ser humano (MACEDO CS, et al., 2017).

Isto se torna mais evidente, quando se trata da pessoa idosa, que naturalmente é acometida por alterações de ordem biológica e psicológica próprias do processo de envelhecimento, que podem ser agravadas na presença de doenças crônicas. Ao atender o idoso, a equipe de saúde deve estar atenta a estas alterações que justificam a oferta de um cuidado diferenciado e interdisciplinar (LIMA TJV, et al., 2010).

Neste sentido, para a construção do PTS o acolhimento, vínculo e corresponsabilização são estratégias centrais (MACEDO CS, et al., 2017). O acolhimento corresponde a uma estratégia para reorganizar o serviço de saúde, redirecionando o olhar ao paciente, reconhecendo suas necessidades singulares, produzindo também espaços de trocas, escuta, manifestação de desejos e necessidades (PAULA VG, et al., 2018).

O vínculo, por sua vez, é compreendido como uma relação contínua no tempo, pessoal, intransferível e calorosa, serve como elo entre paciente/família e a equipe de saúde (BRASIL, 2007). Já a corresponsabilização corresponde a divisão de responsabilidades entre diversos atores, sendo todos responsáveis pelo cuidado e ações (JORGE MSB, et al., 2015).

Sem acolhimento há dificuldade de estabelecer vínculo e sem a criação de vínculo o processo de cuidado do usuário é prejudicado (PAULA VG, et al., 2018).

REAS/EJCH | Vol. Sup. 24 | e659 | DOI: https://doi.org/10.25248/reas.e659.2019 Página 5 de 7 
Neste contexto, a aplicação do PTS com enfoque na saúde do idoso proporciona a integralidade do cuidado e atenção à pessoa idosa com ações direcionadas para esta etapa da vida e/ou situações enfrentadas pelo idoso e que necessitem de intervenções em decorrências de vulnerabilidades identificadas pela equipe.

Reitera-se que para construção do PTS, assim como deste relato, foi preciso compreender a situação do ponto de vista do paciente e de cada membro da equipe multiprofissional, levantar hipóteses diagnósticas que contemplem os aspectos orgânicos, subjetivos e sociais, os riscos e as vulnerabilidades, os limites e as potencialidades dos sujeitos, indo de encontro ao relatado na literatura (BRASIL, 2010; OLIVEIRA GN, 2007).

Além do que, foi necessário levantar as propostas de intervenções e discuti-las com toda a família, buscando a mudança no indivíduo e com ele, uma vez que este precisará de acompanhamento pela equipe de saúde a curto, médio e longo prazo (MACEDO CS, et al., 2017).

Entre as limitações do estudo destaca-se a sobrecarga de responsabilidades assistenciais ocasionada pela alta demanda na unidade, que por vezes, impossibilitava a presença de todos os profissionais nas reuniões, rotatividade da equipe, falta de espaço adequado para discutir o PTS com o usuário e família e formação profissional inadequada para atendimento as necessidades da nova política assistencial, sendo esta limitação considerada um dos principais obstáculos relatado pelos profissionais de saúde.

\section{CONSIDERAÇÕES FINAIS}

Diante do exposto, pode-se constatar que o PTS é uma inovadora ferramenta do cuidado proporcionando aproximação entre equipe, paciente/usuário e família. As atividades desenvolvidas pelos residentes junto à equipe interdisciplinar na construção do PTS contemplam a Política Nacional de Atenção à Saúde do Idoso e integração da teoria na prática cotidiana. Verificou-se que a proposta do PTS permitiu a problematização por parte da equipe multiprofissional das necessidades específicas do idoso, contemplando a integralidade da atenção em uma perspectiva humanizada do atendimento, socialização dos saberes, incorporação da multidimensionalidade nas abordagens e o aprimoramento das práticas assistenciais.

\section{REFERÊNCIAS}

1. BARROS JO. A construção de projetos terapêuticos no campo da saúde mental: apontamentos acerca das novas tecnologias de cuidado. (Dissertação em Ciências) - Faculdade de Fisioterapia, Fonoaudiologia e Terapia Ocupacional da FMUSP, Universidade de São Paulo, 2009.

2. BOCCARDO ACS, et al. O projeto terapêutico singular como estratégia de organização do cuidado nos serviços de saúde mental. Rev. Ter. Ocup. Univ. São Paulo, 2011;22(1):85-92.

3. BRASIL. Portaria Interministerial MEC/MS nํ 1.077, de 12 de novembro de 2009. Dispõe sobre a Residência Multiprofissional em Saúde e a Residência em Área Profissional da Saúde, e institui o Programa Nacional de Bolsas para Residências Multiprofissionais e em Área Profissional da Saúde e a Comissão Nacional de Residência Multiprofissional em Saúde. Diário Oficial da União; Poder Executivo: Brasília, DF, 13 nov. 2009. Seção I, p.7.

4. BRASIL. Ministério da Saúde. Secretaria de Atenção à Saúde. Núcleo Técnico da Política Nacional de Humanização. Clínica ampliada, equipe de referência e projeto terapêutico singular. $2^{\mathfrak{a}}$ ed. Brasília: Ministério da Saúde, 2007. 60 p.

5. BRASIL. Ministério da Saúde. Secretaria de Atenção à Saúde. Política Nacional de Humanização. Atenção Básica. Brasília: Ministério da Saúde, 2010.

6. BRASIL. Ministério da Saúde. Secretaria de Atenção à Saúde Departamento de Ações Programáticas e Estratégicas. Orientações técnicas para a implementação de Linha de Cuidado para Atenção Integral à Saúde da Pessoa Idosa no Sistema Único de Saúde - SUS. Ministério da Saúde, 2018. 91p.

7. BRASIL. Ministério da Saúde. Tabnet. Datasus. Epidemiológicas e Morbidade. 2017. Disponível em: http://datasus.saude.gov.br/. Acesso em: 15 jul. 2018.

8. COELHO LP, et al. Rede de atenção ao idoso: facilitadores e barreiras para implementação. Physis: Revista de Saúde Coletiva, 2018;28(4):e280404. 
9. CUNHA BSS. Envelhecimento e finitude na perspectiva de idosos hospitalizados: contribuições para o cuidado de enfermagem. (Dissertação em Enfermagem) - Universidade Federal Fluminense, Niteroi, 2013; 178p.

10. JORGE MSB, et al. Apoio matricial, projeto terapêutico singular e produção do cuidado em saúde mental. Texto Contexto Enferm, 2015;24(1):112-20.

11. LIMA TJV, et al. Humanização na atenção à saúde do idoso. Saude Soc, 2010;19(4):866-877.

12. MACEDO CS, et al. Elaboração de um projeto terapêutico singular para uma família de alto risco em uma unidade básica de saúde, Macaíba - RN: relato de experiência. Revista Eletrônica Extensão \& Sociedade, 2017;6(1):5166.

13. OLIVEIRA GN. O projeto terapêutico singular. In: Campos GWS, Guerrero AVP, organizadores. Manual de práticas em Atenção Básica: saúde ampliada e compartilhada. Rio de Janeiro: Hucitec; 2007;283-97.

14. PAULA VG, et al. Acolhimento: um olhar inclusivo da Política Nacional de Humanização como estratégia de inclusão social. Revista Educação: Saberes e Práticas, 2018;7(1): 1-10.

15. REIS CS, NORONHA K, WAJNMAN S. Envelhecimento populacional e gastos com internação do SUS: uma análise realizada para o Brasil entre 2000 e 2010. Rev. Bras. Est. Pop, 2016;33(3):591-612.

16. CENSUS BUREAU US. Department of Commerce, 2016. Disponível em: https://www.census.gov/. Acesso em: 08 mar. 2019. 